\title{
HEAT TRANSFER MODELING IN INTEGRATED PHOTOELECTROCHEMICAL HYDROGEN GENERATORS USING CONCENTRATED IRRADIATION
}

\author{
Saurabh Tembhurne ${ }^{1}$, Mikael Dumortier ${ }^{1}$, Sophia Haussener,"* \\ ${ }^{1}$ Laboratory of Renewable Energy Science and Engineering, EPFL, \\ Station 9, 1015 Lausanne, Switzerland
}

\begin{abstract}
The direct conversion of solar energy and water into a storable fuel via integrated photoelectrochemical (PEC) devices is investigated. Particularly, the proposed device uses concentrated solar irradiation in order to minimize the amount of rare and expensive components such as light absorbers and catalysts. Consequently, heat management becomes crucial for device performance. We present a $2 \mathrm{D}$ coupled multi-physics model using finite element and finite volume methods to predict the performance of the integrated PEC device. The model accounts for charge generation and transport in the triple junction solar cell and the components of the integrated electrolyzer (polymeric electrolyte and solid electrode), electrochemical reaction at the catalytic sites, fluid flow and species transport in the channels delivering the reactant (water) and removing the products (hydrogen and oxygen), and radiation absorption and heat transfer in all components. The model developed shows to be a valuable design and optimization tool for integrated PEC devices working with concentrated irradiation and at elevated temperatures.
\end{abstract}

KEY WORDS: Thermal management and control, Numerical simulation, Renewable energy, Cluster Computing, Concentrated multi-junction solar cell

\section{INTRODUCTION}

Photoelectrochemical (PEC) processes constitute a viable route for direct renewable hydrogen production ${ }^{1,2}$. These processes synthesize hydrogen via the electrolysis of water induced by a light generated current on integrated photoactive components. A key issue for economic competitive PEC devices is the reduction of rare and expensive device components, such as catalysts and light absorbers ${ }^{3}$. This can be achieved by concentrating the solar irradiation. However, the performance of the photoactive components is significantly reduced while the ionic transport in the polymeric electrolyte is enhanced with increased temperature until it drops sharply due to membrane dry out ${ }^{4,5,6}$. This coupled behaviour requires a detailed understanding of the heat transfer and, subsequently, the development of heat management strategies via device design and adaptation of the operational conditions.

We propose a highly integrated PEC device composed of a self-tracking concentrator, triple junction solar cell, polymer electrolyte membrane electrolyser, and connecting channels to deliver and remove the reactants and products while preheating the reactants and cooling the temperature-sensitive components. The device is depicted in figure 1 . The concentrator is a glass waveguide, which achieves self-tracking of the sun by a layer of paraffin wax at the bottom of the waveguide. A lens array is used to focus the radiation onto the paraffin wax, the wax is heated by the absorbed infrared radiation, changing the shape of the bottom layer which leads to reflection of light at angles greater than the critical angle for the air-waveguide interface and thus light trapping ${ }^{7}$. The solar irradiation, incident in negative $z$-direction, is concentrated within the waveguide and 
exits the concentrator at its face perpendicular to the $x$-direction. The concentrated radiation is delivered to the triple junction $\mathrm{Ga}_{0.51} \mathrm{In}_{0.49} \mathrm{P} / \mathrm{GaAs} / \mathrm{Si}$ photovoltaic (PV) cell. Its architecture is depicted in figure 1.b. The radiation arriving at the PV cell produces electron-hole-pairs if the radiation energy exceeds the band gap energy of the absorber materials. The holes are delivered to the anode causing the oxidation of the water and the production of oxygen and protons at the catalytic sites. The protons travel to the cathode through the polymeric electrolyte where they are reduced by the electrons delivered form the PV's n-terminal (top contact) to produce hydrogen at the catalytic sites. A water channel between the waveguide exit and the PV cell is introduced to cool the PV cell as well as preheat the reactant (water) before it enters the anodic electrolyser channels. The connection between the water channel and the anodic chamber is represented by black dots in the schematic. The reactant is therefore preheated by the energy which is rejected from the photoabsorbers.

The motivation for this integrated device is the expected increase in efficiency as the longer wavelengths (above the smallest band gap of the solar cell materials), which are usually unused ${ }^{8,9}$, are utilized for the selftracking of the concentrator, and the rejected heat of the PV cell (energy above the band energy which is converted to heat) is utilized for preheating of the reactant.

In order to guide the design and engineering of such an integrated PEC device, we developed a 2D multiphysics model of the PEC device using a commercial finite element solver ${ }^{10}$ coupling local mass and heat transfers for the electrochemical component of the device to the semi-empirical models of the waveguide and detailed multi-physics model of the photovoltaic cell. The simulation domain consists of the $x y$-plane as depicted in figure 1.a. The model will support the development of design and operational guidelines to maximize hydrogen production, energetic efficiencies, durability of the device, and to minimize its size and cost.

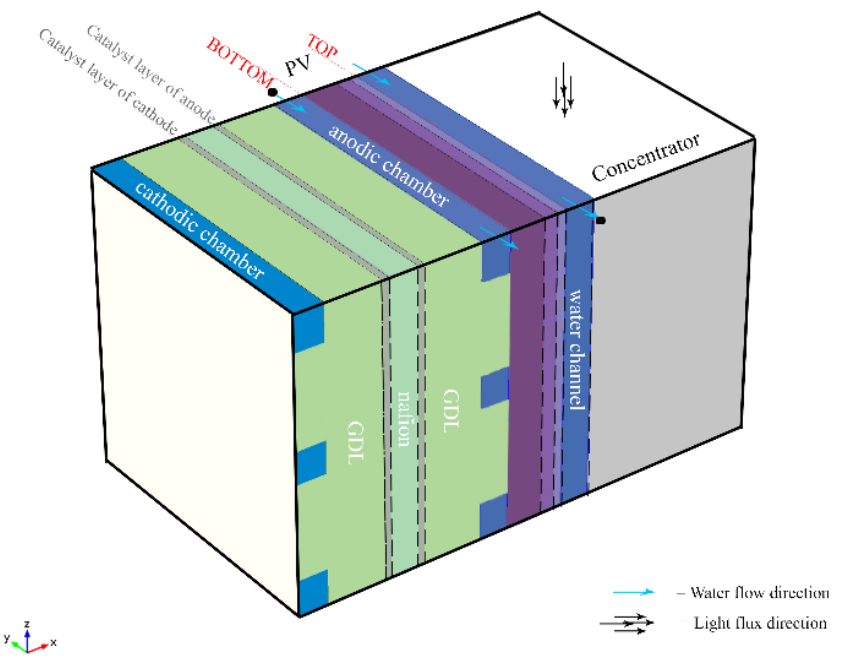

a)

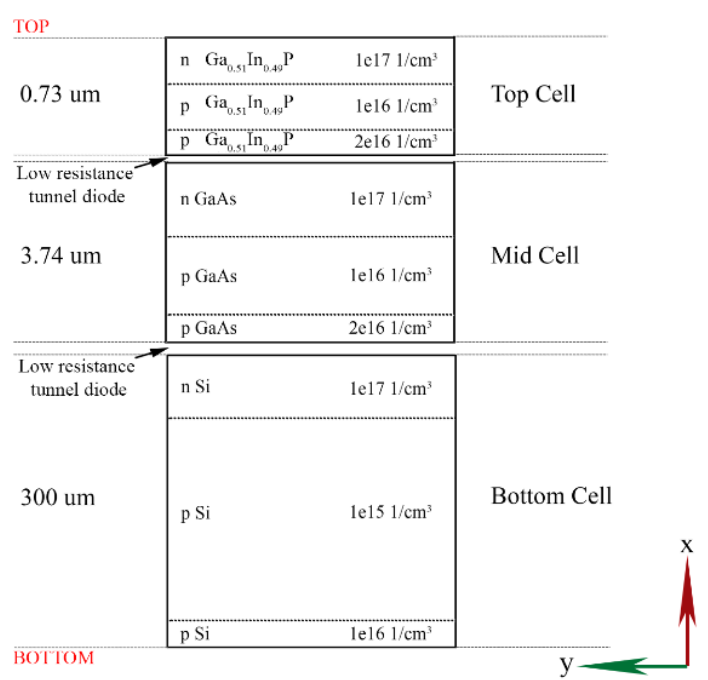

b)

Fig. 1 (a) 3D schematic of the integrated PEC device depicting the self-tracking concentrator, the cooling and preheating water channel, the triple junction solar cell, and the integrated electrolyser consisting of anodic and cathodic channels, gas diffusion layers (GDL), catalyst layers, and polymeric electrolyte (Nafion). (b) Detailed 2D schematic of the triple junction PV cell including the device dimension and doping specifications. The 2D simulation domain is the $x y$-plane. The schematics are not drawn to scale.

\section{METHODOLOGY}

2.1 Electromagnetic wave propagation Eq. (3), which is the combined form of Maxwell curl equations ${ }^{11}$ (1) and (2), is solved via finite element method using the MUltifrontal Massively Parallel Sparse direct Solver (MUMPS) ${ }^{10}$. The $\mathbf{E}$ and $\mathbf{H}$ are solved for a finite number of wavelengths spanning the entire spectral range of the solar irradiation. 


$$
\begin{gathered}
\frac{\partial}{\partial t} \mathbf{B}_{\lambda}(r, t)=-\nabla \times \mathbf{E}_{\lambda}(r, t) \\
\frac{\partial}{\partial t} \mathbf{D}_{\lambda}(r, t)=\nabla \times \mathbf{H}_{\lambda}(r, t)-\mathbf{J}_{\lambda}(r, t) \\
\nabla \times \mu_{r}^{-1}\left(\nabla \times \mathbf{E}_{\lambda}\right)-k_{0}^{2}\left(\varepsilon_{r}-\frac{j \sigma}{\omega \varepsilon_{0}}\right) \mathbf{E}_{\lambda}=0
\end{gathered}
$$

The boundary conditions (BCs) for eq. (3) are depicted in figure 2.a, which consists of irradiation flux BC on the top, and absorbing $\mathrm{BC}$ at the bottom of the simulation domain. The simulation domain is restricted to the water channel and PV regions only as it is assumed that the light doesn't penetrate into the electrochemical cell (EC). The flux at the top boundary is provided by the concentrator's output which is equal to $C \cdot I_{\text {in }}$ where $C$ is the concentration and $I_{\text {in }}$ is the standard AM $1.5 \mathrm{G}$ solar spectrum, i.e. $1000 \mathrm{~W} / \mathrm{m}^{2}$ (assuming no losses in the concentrator). Floquet periodicity is used to account for realistic propagation of the plane wave in $x$ direction. The real part of the calculated electric and magnetic vector fields, $\Re\left(\mathbf{E}_{\lambda}\right)$ and $\Re\left(\mathbf{H}_{\lambda}\right)$, are used to calculate the time averaged Poynting vector ${ }^{11}$, eq. (4), and the corresponding optical generation rate, eq. (5),

$$
\begin{aligned}
\mathbf{S}_{\mathrm{av}_{\lambda}} & =\frac{1}{2} \Re\left(\mathbf{E}_{\lambda} \times \mathbf{H}_{\lambda}\right), \\
G_{\mathrm{opt}_{\lambda}} & =\eta_{\mathrm{opt}} \frac{-\nabla \cdot \mathbf{S}_{\mathrm{av}_{\lambda}}}{h v} .
\end{aligned}
$$

The optical quantum yield, $\eta_{\mathrm{opt}}$, is assumed to be 1 for the photons with energies larger than the band gap of the material. The cut-off wavelength for $\eta_{\text {opt }}$ will be different for each of the three semiconductors of the triple junction $\mathrm{Ga}_{0.51} \mathrm{In}_{0.49} \mathrm{P} / \mathrm{GaAs} / \mathrm{Si}$ solar cell and is determined by the respective band gaps.

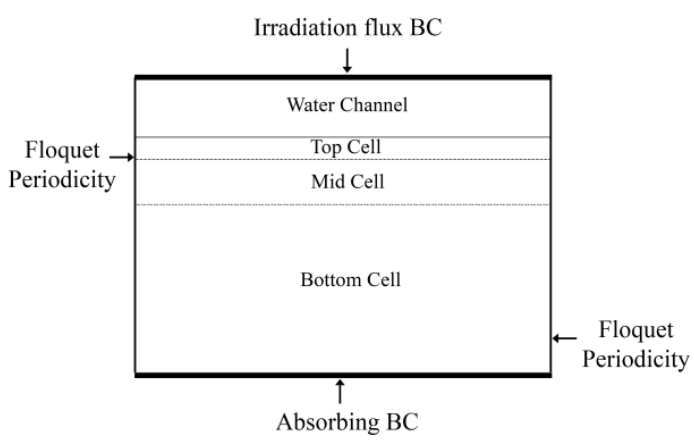

(a)

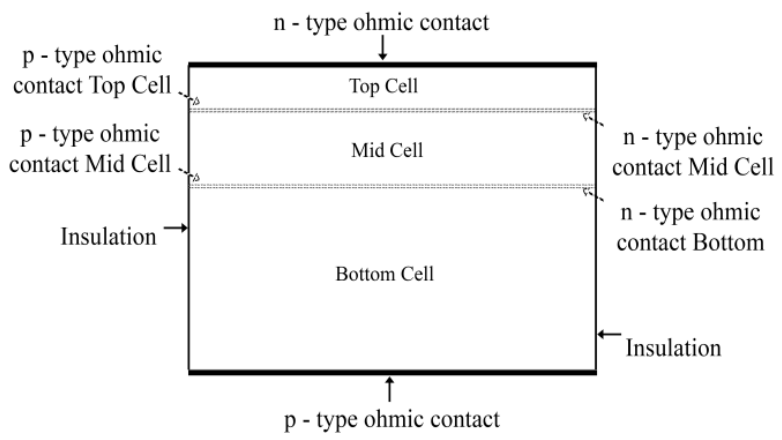

(b)

Fig. 2 Not to scale schematic of the 2D computational domain ( $x y$-plane), indicating the boundary conditions for the solution of (a) the coupled Maxwell equation (eqs. (3)), and (b) the charge generation and transfer equations (eqs. (8) - (10))

The overall generation rate $G_{\mathrm{opt}}$ is the sum of all the individual wavelengths' generation rate, i.e. $\sum_{\lambda} G_{\mathrm{opt}_{\lambda}}$. The total power dissipation density, $Q_{\mathrm{h}}$, is the sum of the electrical (resistive) losses, $Q_{\mathrm{r}}$, and the magnetic losses, $Q_{\mathrm{m}}$, given by:

$$
\begin{gathered}
Q_{\mathrm{r}}=\frac{1}{2}\left(\Re\left(\mathrm{J}_{x} \cdot \mathrm{E}_{x}\right)+\mathfrak{R}\left(\mathrm{J}_{y} \cdot \mathrm{E}_{y}\right)+\mathfrak{R}\left(\mathrm{J}_{z} \cdot \mathrm{E}_{z}\right)\right), \\
Q_{\mathrm{m}}=\Re\left(\frac{1}{2} \mathrm{i} \omega\left(\left(\mathrm{B}_{x} \cdot \mathrm{H}_{x}^{*}\right)+\left(\mathrm{B}_{y} \cdot \mathrm{H}_{y}^{*}\right)+\left(\mathrm{B}_{z} \cdot \mathrm{E}_{z}^{*}\right)\right)\right),
\end{gathered}
$$


where $\mathbf{J}=\sigma \mathbf{E}$ and $\mathbf{B}=\mu \mathbf{H}$. The overall $Q_{\mathrm{h}}$ is calculated from the individual wavelengths' heat dissipation density by summing them together. The concentrator is assumed to be non-absorbing and hence the complete spectrum is available at the outlet of the concentrator.

2.2 Semiconductor charge transport The Poisson equation, eq. (8), and current conservation equations, eqs. (9) and (10), are solved simultaneously, incorporating the definitions of electron current density, $J_{n}$, and hole current density, $J_{p}$, from eqs. (11) and (12), respectively ${ }^{12}$.

$$
\begin{gathered}
\nabla \cdot(\varepsilon \nabla V)=-q\left(p-n+N_{D}^{+}-N_{A}^{-}\right) \\
\nabla \cdot J_{n}=q(R-G)+q \frac{\partial n}{\partial t} \\
-\nabla \cdot J_{p}=q(R-G)+q \frac{\partial p}{\partial t}
\end{gathered}
$$

$\varepsilon$ is the electric permittivity, $q$ is the electronic charge, and $N_{D}^{+}, N_{A}^{-}$are the concentrations of ionized donors and acceptors, respectively, $R$ and $G$ are the carrier recombination and generation rates - including optical generation (eq. (5)).

$$
\begin{gathered}
J_{n}(r, t)=n \mu_{n} \nabla E_{c}+\mu_{n} k_{B} T G\left(n / N_{c}\right) \nabla n+\frac{n q}{T} D_{n, \mathrm{th}} \nabla T, \\
J_{p}(r, t)=p \mu_{p} \nabla E_{v}-\mu_{p} k_{B} T G\left(p / N_{v}\right) \nabla p-\frac{p q}{T} D_{p, \mathrm{th}} \nabla T,
\end{gathered}
$$

$n$ and $p$ are the electron and hole densities, $\nabla E_{c}$ and $\nabla E_{v}$ are the gradients of the local conduction and valence band energies, $T$ is the lattice temperature, $\mu_{n}$ and $\mu_{p}$ are the electron and hole mobilities, and $D_{n, \mathrm{th}}$ and $D_{p, \text { th }}$ are the thermal diffusion coefficients. The function $G$ is defined as $G(\alpha)=\frac{\alpha}{F_{-1 / 2}\left(F_{1 / 2}{ }^{-1}(\alpha)\right)}$ with $F_{-1 / 2}$ as the Fermi-Dirac integral. The total current, and thus its variation with voltage, is obtained from the sum of the hole and electron current densities.

Fermi-Dirac statistics and finite volume solvers ${ }^{10}$ are used to solve eqs. (8) to (10). The BCs for eqs. (8) to (10) are depicted in figure 2.b. They describe the different ohmic contacts used for each part of the PV. Each p-n junction is simulated separately and then the overall PV's current voltage characteristic is generated assuming the series connection of these three p-n junctions. The tunnel diode connecting the two p-n junctions is not modeled assuming that it has negligible losses.

2.3 Heat Transfer The energy conservation equation ${ }^{13}$,

$$
\rho c_{p} \frac{\partial T}{\partial t}+\rho c_{p} \mathbf{u} \cdot \nabla T=\nabla \cdot(k \nabla T)+Q,
$$

is solved in order to calculate the temperature field in the PEC device. Finite element methods and a PARDISO solver ${ }^{10}$ is used to solve eq. (13). $Q=Q_{\mathrm{r}}+Q_{\mathrm{m}}+Q_{\text {react }}$, describes the heat source and it includes the heat from the electromagnetic heating (eqs. (6) - (7)) and chemical reactions ${ }^{14}$ :

$$
Q_{\text {react }}=-J_{\mathrm{s}} \cdot \nabla \phi_{\mathrm{s}}-J_{1} \cdot \nabla \phi_{1}+\left(\phi_{\mathrm{s}}-\phi_{1}-E_{\mathrm{eq}}+T \frac{\partial E_{\mathrm{eq}}}{\partial T}\right) i_{\mathrm{loc}} .
$$


The velocity vector, $\mathbf{u}$, is zero for solid components. The thermal conductivity, $k$, for porous media (e.g. GDL) is replaced by an effective conductivity, $k_{\text {eff }}$, accounting for a volume-averaged electrolyte-solid-conductivity. The BCs for eq. (13) are depicted in figure 3, consisting of thermal insulation on the sidewalls and back side of the simulation domain, and heat flux (natural convection) on the top side. The water channel is supplied initially with water at $293 \mathrm{~K}$ and then it's left to be heated by the incoming radiation and by the rejected PV heat. For the reference case, there is no continuous inflow of the cooling water in the system as the same preheated water is circulated between the anodic channel and the water channel. The concentrator is assumed to be non-absorbing and is modelled by a thermal resistance approach ${ }^{13}$, thus, $h_{\text {eff }}=\frac{1}{\left(1 / h_{\text {coeff }}\right)+\left(L_{\text {conc }} / k_{\text {conc }}\right)}$ with $L_{\text {conc }}$ representing the mean photon flux length in the concentrator.

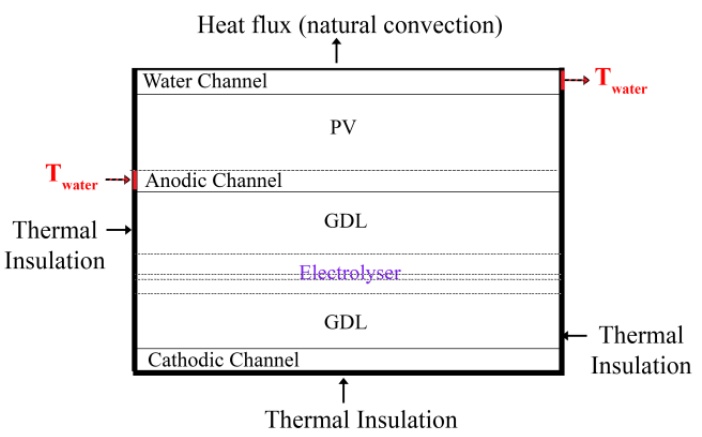

Fig. 3 Not to scale schematic of the 2D computational domain ( $x y$-plane), indicating the boundary conditions for the solution of the energy equation, eq. (13).

2.4 Electrolyser charge transport and reacting flow Charge transport in the electrode and electrolyte $(k$ $=1, \mathrm{~s}$, respectively, for ionic or electronic conductors) is given by ${ }^{14}$

$$
\begin{gathered}
\nabla \cdot \mathbf{J}_{k}=Q_{k}, \\
\mathbf{J}_{k}=-\sigma_{k} \nabla \phi_{k},
\end{gathered}
$$

accounting for the electrochemical reaction via the reaction current, $i_{\text {loc }}$, modeled via Butler-Volmer expression $^{14}$,

$$
i_{\text {loc }, m}=i_{0, m}\left(\mathrm{C}_{\mathrm{R}} \exp \left(\frac{\alpha_{\mathrm{a}, m} F \eta_{\mathrm{act}, m}}{R T}\right)-C_{\mathrm{O}} \exp \left(\frac{-\alpha_{\mathrm{c}, m} F \eta_{\mathrm{act}, m}}{R T}\right)\right),
$$

where $\eta_{\text {act }, m}$ is the activation overpotential for reaction $\mathrm{m}$ and the total overpotential of the reaction is given by

$$
\eta_{m}=\phi_{\mathrm{s}}-\phi_{1}-E_{\mathrm{eq}, m},
$$

for $m=1,2$, accounting for the anodic one-step oxygen evolution reaction (OER),

$$
2 \mathrm{H}_{2} \mathrm{O} \rightarrow \mathrm{O}_{2}+4 \mathrm{H}^{+}+4 \mathrm{e}^{-}
$$

and cathodic one-step hydrogen evolution reaction,

$$
4 \mathrm{H}_{2} \mathrm{O}+4 \mathrm{e}^{-} \rightarrow 2 \mathrm{H}_{2}+4 \mathrm{OH}^{-}
$$

The charge conservation equations, eqs. (15) - (16), are solved via finite element methods and a MUMPS solver ${ }^{10}$. The boundary conditions for eqs. (15) - (16) are depicted in figure 4.a, where a positive electric potential is applied to the anode side and the cathode is maintained at zero potential with insulation on the sidewalls of the simulation domain. 
The fluid flow and mass transport in the channels and the porous gas diffusion layers are modelled by the Navier-Stokes equations with the Darcy extension ${ }^{15,16}$,

$$
\frac{\rho}{\varepsilon_{p}}(\mathbf{u} \cdot \nabla) \frac{\mathbf{u}}{\varepsilon_{p}}=\nabla \cdot\left[-\mathrm{P} \cdot \mathrm{I}_{d}+\frac{\mu_{d}}{\varepsilon_{p}}\left(\nabla \mathbf{u}+(\nabla \mathbf{u})^{T}\right)-\frac{2}{3} \frac{\mu_{d}}{\varepsilon_{p}}(\nabla \cdot \mathbf{u}) \mathrm{I}_{\mathrm{d}}\right]-\left(\frac{\mu_{d}}{\kappa_{p}}+\frac{Q_{\mathrm{br}}}{\varepsilon_{p}{ }^{2}}\right) \mathbf{u}+\mathbf{F},
$$

and the species transport (eq. (22)) is modelled by the Maxwell-Stefan diffusion model ${ }^{17}$ for the low density fluid mixture, with the diffusivities replaced by the binary diffusivities for the present species pairs,

$$
\rho \frac{\partial}{\partial t}\left(\omega_{i}\right)+\rho(\mathbf{u} \cdot \nabla) \omega_{i}=-\nabla \cdot \mathbf{j}_{i}+R_{i}
$$

where $\omega_{i}$ is the mass fraction and $\mathbf{j}_{i}$ is the mass flux relative to the mass average velocity $\mathbf{u}$ given by ${ }^{18}$

$$
\mathbf{j}_{i}=-\rho \omega_{i} \sum_{k} D_{i k} \mathbf{d}_{k}-D_{i}^{T} \frac{\nabla T}{T}
$$

$R_{i}$ is the rate expression describing its production or consumption, $D_{i k}$ are the multicomponent Fick diffusivities, $D_{i}^{T}$ are the thermal diffusion coefficients, and $\mathbf{d}_{k}$ is the diffusional driving force acting on species $k$. The species transport equation, eq. (22), are solved via finite element methods and a MUMPS solver ${ }^{10}$. BCs for eq. (22) are depicted in figure 4.b, describing the inlet and outlet conditions of the different channels. The water at the output of the water channel is fed to the anodic channel at its inlet with normal velocity, $v_{\text {water. }}$ There is no continuous inflow of cooling water to water channel; the same water, initially supplied in the water channel, is circulated between the anodic channel and the top water channel.

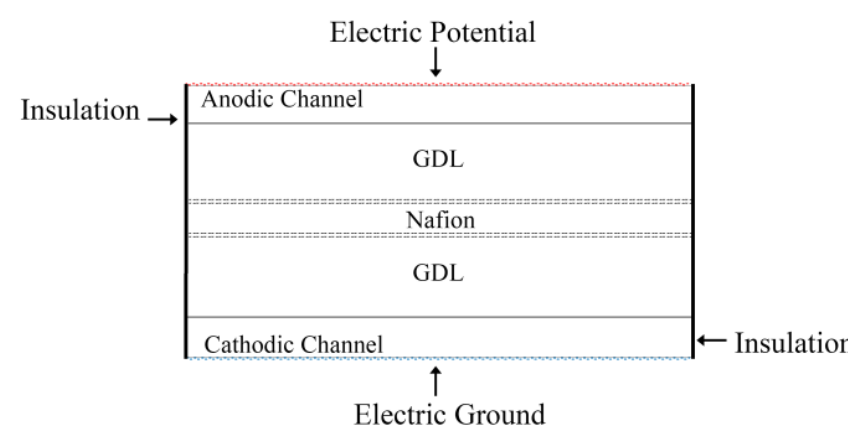

(a)

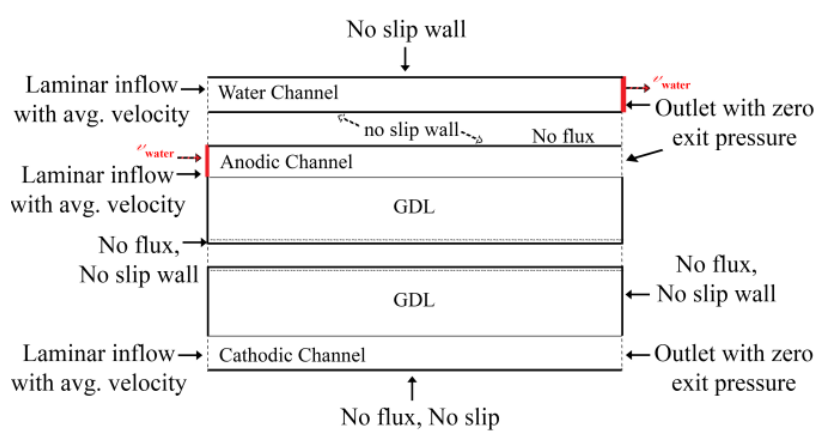

(b)

Fig. 4 Schematic ( $x y$-plane) showing boundary conditions for (a) electrochemical simulation, and (b) fluid flow (both reactive and non-reactive flow) simulations. (Note: Schematic is not drawn to scale).

2.5 Meshing strategy and computational expense Different meshing strategies are adopted for different physical simulation modules in order to minimize the overall solution time of the coupled multi-physics problem and assure mesh independence of the solution. The most restrictive requirement came from the EM simulations (eqn. (3)) as minimum element size in the direction of incident radiation has to be at least $1 / 5$ of the incoming wavelength. Additionally, this minimum element size is adjusted by a factor of $1 / n_{\text {ref }}$ to take into account the change in the refractive index as light travels from one material to another. A variable mesh approach was used for different wavelengths to minimize the computing time. The solution of the other transport and conservation equations, eqs. (8) - (10), (13), (15) - (18), were similarly optimized in order to allow for mesh convergence and to minimize the computational expense. Particularly, boundary meshes with minimum wall distance given by the "Y+ wall distance estimation method" were used to resolve the boundary layer for fluid flow, mass and heat transfer calculations. The distributed mesh was used to get a dense mesh near the boundary layer and a coarser mesh in the center of the fluid channel. A meshing approach with smaller element sizes, i.e. $8.3 \mathrm{~nm} \times 30 \mu \mathrm{m}$ in domain dimension of $100 \mathrm{~nm} \times 1 \mathrm{~cm}$, was used in the anode and cathode layers, where the electrochemical reaction 
occurs. The mesh size was increased in the other domains for the solution of eqs. (9) to (18) in order to reduce computational time. A workstation with 64GB RAM and 8 cores was used to solve the coupled equations. It needed 16 hours to solve one global iteration (see following section).

2.6 Simulation flow Figure 5 depicts the flow diagram of the coupled model containing six physical modules: EM, HT, FF, RFF, EC, SD. The EM simulation is performed for the given temperature field, starting with a constant initial temperature $\left(T_{\text {init }}=293 \mathrm{~K}\right)$. The resulting heat dissipation density is input to the heat transfer $(\mathrm{HT})$ simulation module. The interaction between different simulation modules is shown by the respective arrows. The fluid flow (FF) module provides the velocity of the water, flowing in the anodic channel, to the reacting fluid flow (RFF) module and the heat transfer (HT) module. The HT module provides the temperature input to all the other modules. Being fed by water concentration from the RFF module and temperature from the HT module, the EC module provides $Q_{\text {react }}$ to HT and $i_{\text {loc }}$ to RFF. In parallel, the semiconductor device simulation is performed starting with $T_{\text {init. }}$. The resulting current densities and electric potential from the semiconductor and electrolyser charge transfer equations are input to the operating point calculation module, which calculates the operating current and voltage. If the PV temperature distribution, $T_{\mathrm{op}}$, at the operating point is not equal to the initial temperature of the semiconductor simulation module $\left(T_{\text {semi }}\right)$, then the new $T_{\text {op }}$ is provided to the corresponding modules and the loop is repeated. This process is repeated iteratively until the temperature converges $\left(T_{\mathrm{op}, \text { old }}-T_{\mathrm{op}, \text { new }}<\varepsilon_{\mathrm{r}}\right)$.

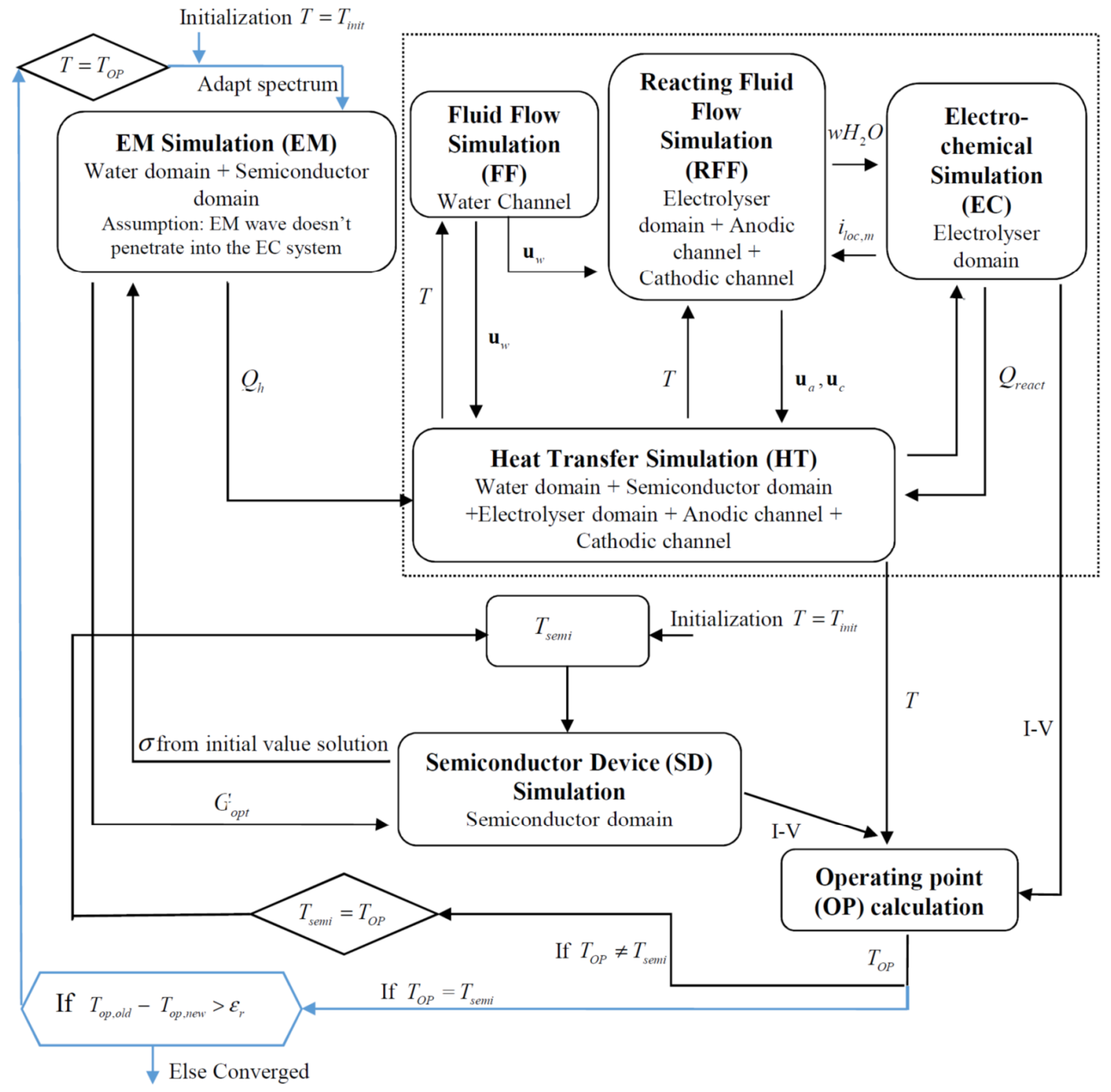

Fig. 5 The simulation flow of the Integrated PEC system. 
IHTC15-9526

\section{REFERENCE CASE}

The simulation results for a reference case are given. The dimensions and component characteristics of the reference case are given in Tables 1 and 2.

Table 1 Temperature independent baseline parameters used in the simulation for the reference case.

\begin{tabular}{lll}
\hline \hline Parameter name & Parameter value & Unit \\
\hline Irradiation concentration $(C)$ & 1 & - \\
No. of wavelengths for EM simulation & 100 & - \\
Concentrator thickness & 5 & $\mathrm{~cm}$ \\
Integrated PEC system width (PV+EC) & 1.00 & $\mathrm{~cm}$ \\
Integrated PEC system depth (PV+ EC) & 1.755 & $\mathrm{~mm}$ \\
Diameter of water channel on top of PV & 0.0002 & $\mathrm{~m}$ \\
Thickness of Ga $0.51 \mathrm{In}_{0.49} \mathrm{P}$ & 0.73 & $\mu \mathrm{m}$ \\
Thickness of GaAs & 3.74 & $\mu \mathrm{m}$ \\
Thickness of Si & 300 & $\mu \mathrm{m}$ \\
Diameter of Anodic and Cathodic channels of EC & 0.0002 & $\mathrm{~m}$ \\
Thickness of GDL & 400 & $\mu \mathrm{m}$ \\
Thickness of Catalyst layer & 100 & $\mathrm{~nm}$ \\
Thickness of nafion/membrane & 50.8 & $\mu \mathrm{m}$ \\
HER anodic transfer coefficient & 1 & - \\
HER cathodic transfer coefficient & 1 & - \\
OER anodic transfer coefficient & 1.7 & - \\
OER cathodic transfer coefficient & 0.1 & - \\
\hline \hline
\end{tabular}

Table 2 Temperature dependent baseline parameters used in the simulation for the reference case.

\begin{tabular}{|c|c|c|}
\hline Parameter name & Parameter value & Unit \\
\hline$E_{\mathrm{g}}$ of $\mathrm{Ga}_{0.51} \mathrm{In}_{0.49} \mathrm{P}$ & $1.8773^{*}$ & \\
\hline$E_{\mathrm{g}}$ of $\mathrm{GaAs}^{19}$ & $1.519-\frac{5.41 \times 10^{-4} T^{2}}{T+204}$ & $\mathrm{eV}$ \\
\hline$E_{\mathrm{g}}$ of $\mathrm{Si}^{19}$ & $1.166-\frac{4.73 \times 10^{-4} T^{2}}{T+636}$ & $\mathrm{eV}$ \\
\hline $\begin{array}{l}\text { Electron (Hole) mobility using the Arora } \\
\text { Mobility Model }{ }^{20}\end{array}$ & 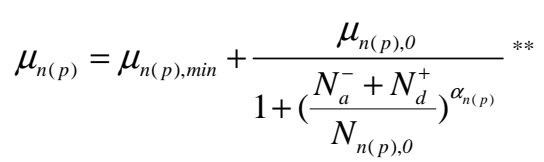 & $\mathrm{m}^{2} /(\mathrm{Vs})$ \\
\hline Electrical conductivity of Nafion ${ }^{21,22}$ & $22.73 \times \exp \left(-\frac{2000}{R T}\right)$ & $\mathrm{S} / \mathrm{m}$ \\
\hline Anodic exchange current density $23,24,25$ & $4.62 \times \exp \left(-\frac{48600}{R T}\right)$ & $\mathrm{A} / \mathrm{cm}^{2}$ \\
\hline Cathodic exchange current density ${ }^{26,27}$ & $142.02 \times \exp \left(-\frac{28900}{R T}\right)$ & $\mathrm{A} / \mathrm{cm}^{2}$ \\
\hline $\begin{array}{l}\text { Thermal conductivity, } \\
\text { Specific heat capacity at constant pressure and } \\
\text { volume, Density of } \mathrm{H}_{2} \mathrm{O}, \mathrm{H}_{2}, \mathrm{O}_{2}, \mathrm{Ar}\end{array}$ & Interpolated ${ }^{28}$ & \\
\hline
\end{tabular}

*The band gap of Ga0.51 In0.49P is assumed to be constant (due to lack of sufficient available data) only for this reference case where irradiation concentration is 1 and we assume this would not lead to large temperature variations. However when working with concentrations $>1$, it is necessary to include temperature dependence of $\mathrm{Ga}_{0.51} \operatorname{In}_{0.49} \mathrm{P}$.

** For the reference case, we considered the mobility's temperature variation only for $\mathrm{Si}$ as it is the current limiting semiconductor and hence would capture the majority of the effect. 
The AM 1.5G solar irradiation spectrum was modified to contain 100 equidistant wavelengths between $280 \mathrm{~nm}$ - $2500 \mathrm{~nm}$, as depicted in figure 6 , in order to reduce the number of wavelengths to be simulated but still to capture the spectral variations in the visible and infrared parts of the spectrum.

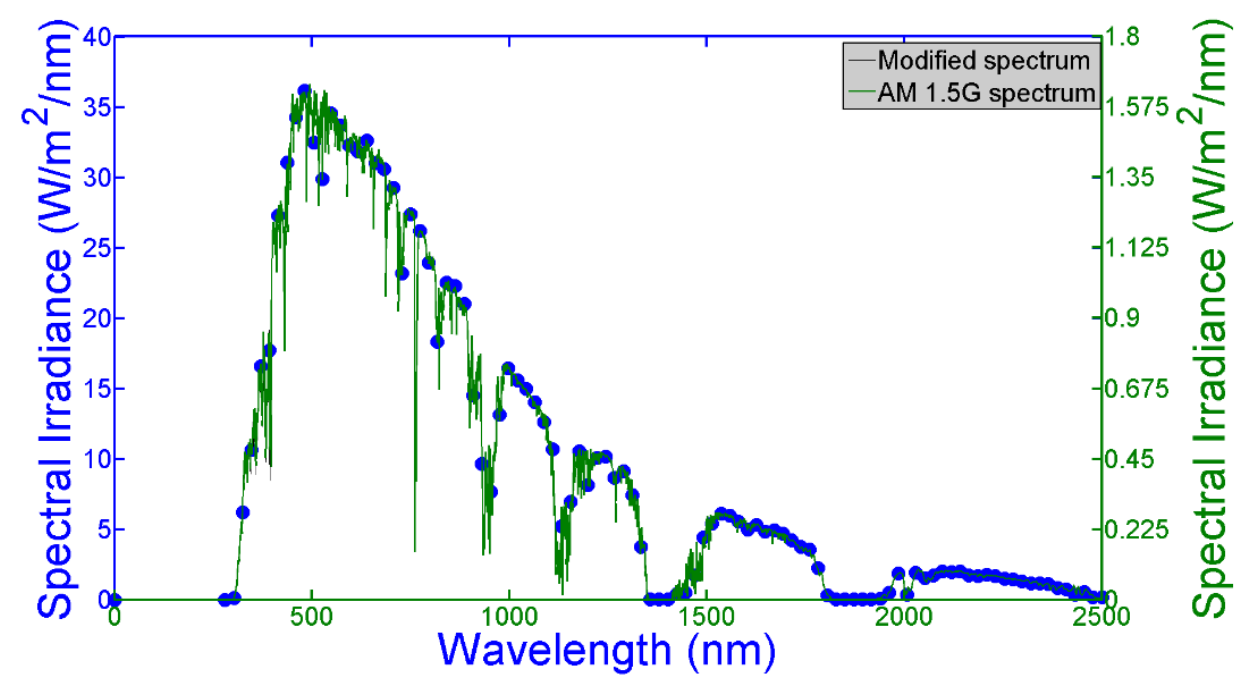

Fig. 6 Modified (blue) and original (green) 1.5 AM solar spectrum.

Figure 7.a depicts the heat dissipation density inside the electrolyser. The heat is generated only in the anode and cathode regions, accounting for the water electrolysis reaction being endothermic. The generation rate and the EM heat dissipation density at the final operating temperature are shown in figures 7.b and 7.c. The generation rate and the electromagnetic heat dissipation density exhibit their peaks close to the top boundary of the PV. This results from the large extinction for different PV materials. The peak of heat dissipation density is observed within the material (at $x=1.39 \mu \mathrm{m}$ ) as the longer wavelengths have a smaller extinction coefficient.

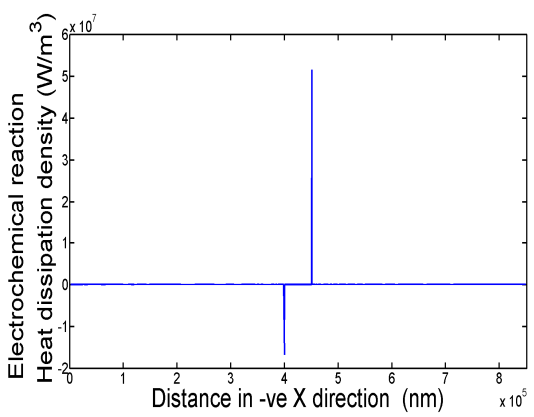

(a)

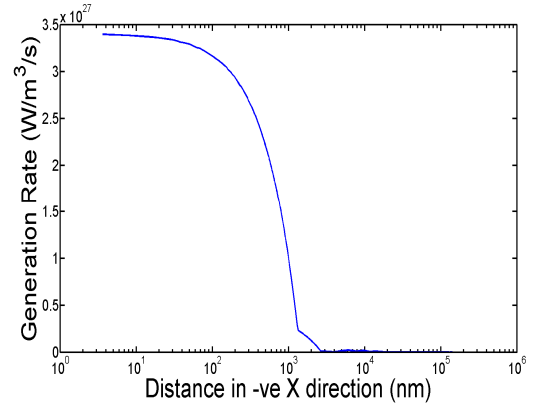

(b)

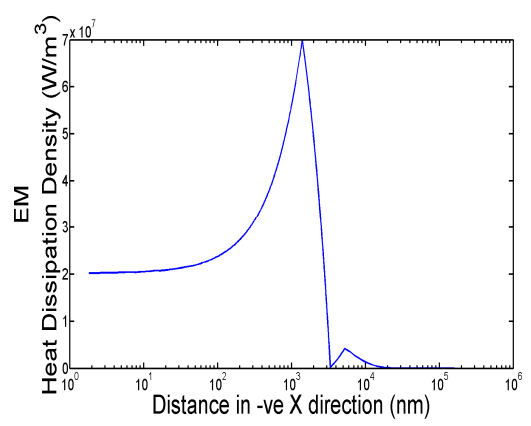

(c)

Fig. 7 (a) Heat dissipation density at the operating point inside EC. The left side is the top of EC which is close to the PV and hence downward peak corresponds to anode and upward peak to cathode. (b) Generation rate and (c) EM heat dissipation density inside the complete PEC device at the operating point; the left side corresponds to the top of the PV which is adjacent to the concentrator.

The variation of the generation rate and the EM heat dissipation density with temperature, for $C=1$, is minimal, leading to very small variations in operating temperature $(0.8 \%)$. Nevertheless, the operational temperature for $C=1$ is several degrees above the room temperature, leading to a noticeable change in the performance of the PV. With increase in temperature under standard AM1.5G illumination without radiation concentration, the band gap and the open circuit voltage of the semiconductors decreases, leading to an increased range of absorbable wavelengths. With greater spectral response of the PV, the number of wavelengths contributing to heat dissipation decreases and a decrease in heat dissipation density. Overall, we see an increase in the short circuit current, decrease in the open circuit voltage, and decrease in the operating temperature. 
Figure 8 shows the intersection of EC and PV performance curves and thus the calculation of the operating point. The intersection points lie well in the plateau region of the PV's performance curve and hence the operating voltage is independent of the variations in temperature of the PV. However as the PV's current density increases with temperature the intersection point moves vertically higher (see figure 8) leading to higher value of the operating current density and hence higher hydrogen production.

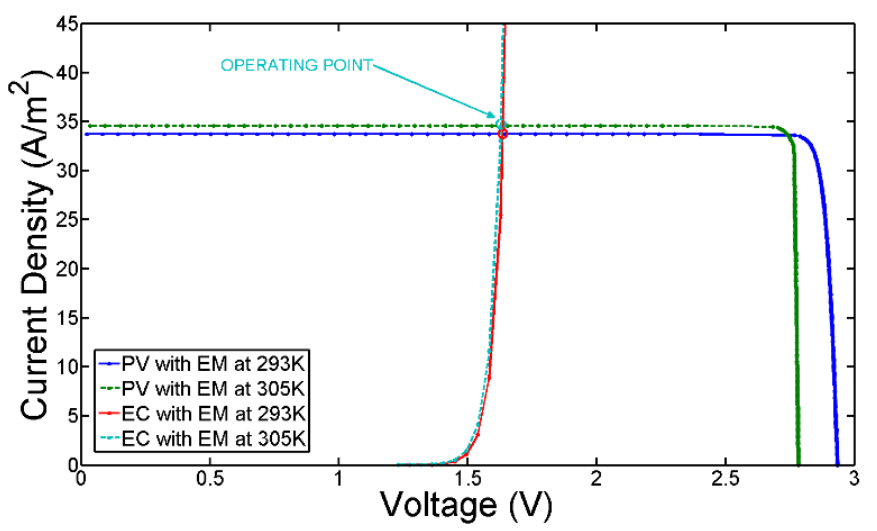

Fig. 8 Current density versus potential of the triple junction PV and the EC at 293K and 305K.

The change in absorbable spectrum with temperature requires the choice of an adaptive wavelength spectrum for each iteration step. The minimum wavelength difference constituting the spectrum is given by eq. (24) when considering a temperature change from $T_{1}$ to $T_{2}$,

$$
\frac{h c}{E_{\mathrm{g}}(0)-\frac{\alpha T_{1}^{2}}{T_{1}+\beta}}-\frac{h c}{E_{\mathrm{g}}(0)-\frac{\alpha T_{2}^{2}}{T_{2}+\beta}}=\Delta \lambda_{\min } .
$$

The mobility of electrons and holes in the semiconductor is significantly influenced by the temperature. We used the Arora mobility model ${ }^{20}$. Both mobilities decrease with temperature leading to an increase in resistivity and increased heat dissipation. As this effect is contrary to the effect of band gap variation discussed earlier, only a coupled model can predict the true temperature profile of the integrated PEC system.

The solar to hydrogen efficiency ( STH ) is calculated using

$$
\mathrm{STH}=\frac{J_{\mathrm{OP}} \cdot V_{\mathrm{OP}}}{C \cdot P_{\mathrm{in}}},
$$

assuming Faradaic efficiencies of 1 and negligible product crossover. The STH for the reference case was calculated to be $5.67 \%$. It is anticipated that with increased optical concentration the current density provided by the PV and the temperature of water feeding the EC would increase, both contributing to an enhanced STH. Nevertheless, charge transfer resistances in the semiconductor and electrodes would increase, reducing the STH.

\section{CONCLUSION}

We developed a coupled multi-physics model and solution methodology to simulate the performance of integrated photoelectrochemical devices using concentrated solar irradiation. The model couples electromagnetic wave propagation, semiconductor charge generation and transport, heat transfer, fluid flow, mass transport, electrolyte and electrode charge transport, and electrochemical reactions. Finite element and finite volume methods were used to solve the governing equations and the corresponding boundary conditions. 
Complex temperature dependencies were included in different physics' models. The absorbable spectrum changed with temperature and hence required an adaptive spectrum changing for each iteration step, giving rise to a trade-off between precision and computation time.

The peak of electron hole pair generation and electromagnetic heat dissipation density lied close to the top boundary of the PV mainly due to the large extinction coefficient of the triple junction PV's materials. The heat generation inside the electrolyser existed only in the cathode and anode region. The integrated PEC device exhibited minimal ohmic losses mainly due to the large conductivity of the electrodes and the small ionic path lengths in the thin electrolyte. Consequently, the operating point of the integrated system lied in the plateau region of the triple junction PV's performance curve, which exhibited an unnecessary large open circuit potential. Consequently, the operating voltage showed to be independent of the variations in temperature. However, the operating current density increased with increasing temperature as the PV's current density increased with temperature and, consequently, led to enhanced hydrogen production. This effect showed to be dominating the negative temperature effects due to decreased mobility, increased resistivity, and increased heat dissipation.

The model developed shows promise to be a valuable design and optimization tool for PEC cells working with concentrated irradiation and at elevated temperatures.

\section{ACKNOWLEDGMENT}

We acknowledge the financial support of the Swiss National Science Foundation via Project SHINE (contract number 20NA21_145936). We thank Fouad Aabid and Yannick Gaudy for fruitful discussions.

\section{NOMENCLATURE}

\begin{tabular}{|c|c|c|c|c|c|}
\hline$\alpha_{\mathrm{a}}$ & Anodic charge transfer & & $\mathbf{H}$ & Magnetic field intensity & $(\mathrm{A} / \mathrm{m})$ \\
\hline$\alpha_{\mathrm{c}}$ & $\begin{array}{l}\text { coefficient } \\
\text { Cathodic charge transfer }\end{array}$ & $(-)$ & $i_{\mathrm{loc}}$ & $\begin{array}{l}\text { Local charge transfer current } \\
\text { density }\end{array}$ & $\left(\mathrm{A} / \mathrm{m}^{2}\right)$ \\
\hline & coefficient & $(-)$ & $i_{0}$ & Exchange current density & $\left(\mathrm{A} / \mathrm{m}^{2}\right)$ \\
\hline B & Magnetic flux density & (T) & $I_{\text {in }}$ & Incident flux & $\left(\mathrm{W} / \mathrm{m}^{2}\right)$ \\
\hline D & Electric flux density & $\left(\mathrm{C} / \mathrm{m}^{2}\right)$ & $I_{A}$ & Identity matrix & $(-)$ \\
\hline$D_{i k}$ & Fick diffusivities & & $\mathbf{J}^{a}$ & Current density & $\left(\mathrm{A} / \mathrm{m}^{2}\right)$ \\
\hline $\mathbf{d}_{k}$ & Diffusional force acting on & & $J_{n}$ & Electron current density & $\left(\mathrm{A} / \mathrm{m}^{2}\right)$ \\
\hline & $\begin{array}{l}\text { species k } \\
\text { Thermal diffusion coeff. }\end{array}$ & $\begin{array}{l}(-) \\
(\mathrm{kg} /(\mathrm{m}\end{array}$ & $J_{O P}$ & Operating current density & $\left(\mathrm{A} / \mathrm{m}^{2}\right)$ \\
\hline & $\begin{array}{l}\text { Thermal diffusion coeff. } \\
\text { Electron thermal diffusion coef }\end{array}$ & $\begin{aligned} & (\mathrm{kg} /(\mathrm{m} \mathrm{s})) \\
= & (\mathrm{kg} /(\mathrm{m} \mathrm{s}))\end{aligned}$ & $J_{p}$ & Hole current density & $\left(\mathrm{A} / \mathrm{m}^{2}\right)$ \\
\hline$D^{\prime}$ & Hole thermal diffusion coeff & & $J_{1}$ & Electrolyte current density & $\left(\mathrm{A} / \mathrm{m}^{2}\right)$ \\
\hline $\mathbf{E}$ & Electric field intensity & $(\mathrm{kg} /$ & $J_{\mathrm{s}}$ & Electrode current density & $\left(\mathrm{A} / \mathrm{m}^{2}\right)$ \\
\hline$E_{\mathrm{eq}}$ & Equilibrium potential & (V) & $k_{\mathrm{conc}}$ & Thermal conductivity of conc. & $(\mathrm{W} /(\mathrm{m}$ \\
\hline$E_{\mathrm{ph}}$ & Photon energy & $(\mathrm{eV})$ & $\kappa_{p}$ & $\begin{array}{l}\text { Permeability of the porous } \\
\text { medium }\end{array}$ & $\left(\mathrm{m}^{2}\right)$ \\
\hline $\begin{array}{l}\varepsilon \\
\varepsilon_{p}\end{array}$ & $\begin{array}{l}\text { Electric permittivity } \\
\text { Porosity }\end{array}$ & $\begin{array}{l}(\mathrm{F} / \mathrm{m}) \\
(-)\end{array}$ & $L_{\text {conc }}$ & Length of Concentrator through & \\
\hline$\varepsilon_{r}$ & Convergence error & $(-)$ & & flow length & $(\mathrm{m})$ \\
\hline$F$ & Faraday's constant & $\left(\mathrm{A} \mathrm{s} \mathrm{mol}{ }^{-1}\right)$ & $n$ & Electron density & $\left(1 / \mathrm{m}^{3}\right)$ \\
\hline$G_{\mathrm{opt}}$ & Optical Generation rate & $\left(\mathrm{W} / \mathrm{m}^{3} / \mathrm{s}\right)$ & $N_{a}^{-}$ & Ionized acceptor concentration & $\left(1 / \mathrm{m}^{3}\right)$ \\
\hline$h$ & Planck constant & $(\mathrm{J} \mathrm{s})$ & $N_{d}^{+}$ & Ionized donor concentration & $\left(1 / \mathrm{m}^{3}\right)$ \\
\hline
\end{tabular}




\begin{tabular}{|c|c|c|c|c|c|}
\hline$N_{c}$ & Effective density of states in & & $\begin{array}{l}\text { STH } \\
\sigma\end{array}$ & $\begin{array}{l}\text { Solar to Hydrogen efficiency } \\
\text { Electrical conductivity }\end{array}$ & $\begin{array}{l}(-) \\
(\mathrm{S} / \mathrm{m})\end{array}$ \\
\hline$N_{v}$ & $\begin{array}{l}\text { Conduction band } \\
\text { Effective density of states in }\end{array}$ & $\left(1 / \mathrm{m}^{3}\right)$ & $\begin{array}{l}\sigma \\
T_{\text {init }}\end{array}$ & Initial temperature & $(\mathrm{K})$ \\
\hline & Valence band & $\left(1 / \mathrm{m}^{3}\right)$ & $T_{l}$ & Lattice temperature & $(\mathrm{K})$ \\
\hline$N_{n(p), 0}$ & $\begin{array}{l}\text { Reference impurity parameter } \\
\text { for electrons (holes) }\end{array}$ & $\left(1 / \mathrm{m}^{3}\right)$ & $T_{\mathrm{OP}}$ & $\begin{array}{l}\text { Operating temperature of } \mathrm{PV} \\
\text { /semiconductor }\end{array}$ & $(\mathrm{K})$ \\
\hline$n_{\text {ref }}$ & Refractive index & $(-)$ & $\mathbf{u}$ & Velocity vector & $(\mathrm{m} / \mathrm{s})$ \\
\hline$\eta_{\text {act }, m}$ & Activation overpotential & $(\mathrm{V})$ & $\mathbf{u}_{\mathrm{w}}$ & Velocity field in water channel & $(\mathrm{m} / \mathrm{s})$ \\
\hline$\eta_{\mathrm{opt}}$ & $\begin{array}{l}\text { corresponding to reaction } \mathrm{m} \\
\text { Quantum yield }\end{array}$ & $(-)$ & $\begin{array}{l}\mathbf{u}_{\mathrm{a}} \\
\mathbf{u}_{\mathrm{c}}\end{array}$ & $\begin{array}{l}\text { Velocity field in anodic channel } \\
\text { Velocity field in cathodic }\end{array}$ & $(\mathrm{m} / \mathrm{s})$ \\
\hline$\eta_{m}$ & Total overpotential correspondir & ng to & & Channel & $(\mathrm{m} / \mathrm{s})$ \\
\hline & reacti & $(\mathrm{V})$ & $\mu$ & Magnetic permeability & $(\mathrm{H} / \mathrm{m})$ \\
\hline$P$ & Pressure & (Pa) & $\mu_{d}$ & Dynamic viscosity & $(\mathrm{kg} /(\mathrm{m} \mathrm{s}))$ \\
\hline$P_{\text {in }}$ & AM1.5G input intensity & $\left(\mathrm{W} / \mathrm{m}^{2}\right)$ & $\mu_{n}$ & Electron mobility & $\left(\mathrm{m}^{2} /(\mathrm{V} \mathrm{s})\right)$ \\
\hline$p$ & Hole density & $\left(1 / \mathrm{m}^{3}\right)$ & $\mu_{p}$ & Hole mobility & $\left(\mathrm{m}^{2} /(\mathrm{V} \mathrm{s})\right)$ \\
\hline$\rho$ & Density & $\left(\mathrm{kg} / \mathrm{m}^{3}\right)$ & $\mu_{n(p), \min }$ & Electron (hole) mobility & \\
\hline$\phi_{1}$ & Electrolyte potential & $(\mathrm{V})$ & & reference minimum & $\left(\mathrm{m}^{2} /(\mathrm{V} \mathrm{s})\right)$ \\
\hline$\phi_{\mathrm{s}}$ & Electrode potential & $(\mathrm{V})$ & $\mu_{n(p), 0}$ & Electron (hole) mobility & \\
\hline$Q_{\mathrm{br}}$ & Mass source or mass sink & $\left(\mathrm{kg} /\left(\mathrm{m}^{3} \mathrm{~s}\right)\right)$ & & reference & $\left(\mathrm{m}^{2} /(\mathrm{V} \mathrm{s})\right)$ \\
\hline$Q_{\mathrm{h}}$ & Total heat dissipation density & $\left(\mathrm{W} / \mathrm{m}^{3}\right)$ & $V_{\mathrm{OP}}$ & Operating voltage & $(\mathrm{V})$ \\
\hline$Q_{\mathrm{m}}$ & Magnetic losses & $\left(\mathrm{W} / \mathrm{m}^{3}\right)$ & $v$ & Frequency of incoming light & $(\mathrm{Hz})$ \\
\hline$Q_{\mathrm{r}}$ & Resistive losses & $\left(\mathrm{W} / \mathrm{m}^{3}\right)$ & $\omega_{i}$ & Mass fraction & $(-)$ \\
\hline$Q_{\text {react }}$ & $\begin{array}{l}\text { Heat source from chemical } \\
\text { reactions }\end{array}$ & $\left(\mathrm{W} / \mathrm{m}^{3}\right)$ & $\omega$ & $\begin{array}{l}\text { Angular frequency of incoming } \\
\text { Light }\end{array}$ & $(\mathrm{rad} / \mathrm{s})$ \\
\hline S & Time averaged Poynting vector & $\left(\mathrm{W} / \mathrm{m}^{2}\right)$ & & & \\
\hline
\end{tabular}

\section{REFERENCES}

[1] M. Gratzel, Photoelectrochemical cell, Nature, 414, 2001.

[2] Z. Li, W. Luo, M. Zhang, J. Fenga and Z. Zoua, Photoelectrochemical cells for solar hydrogen production: current state of promising photoelectrodes, methods to improve their properties and outlook, Energy Environ. Sci., 6, 2013.

[3] B.A. Pinaud et al. Technical and economic feasibility of centralized facilities for solar hydrogen production via photocatalysis and photoelectrochemistry, Energy Environ. Sci., 6, 2013.

[4] S. Haussener et al. Simulations of the irradiation and temperature dependence of the efficiency of the tandem photoelectrochemical water-splitting systems, Energy Environ. Sci., 2013.

[5] P. Singh and N. Ravindra, Temperature dependence of solar cell performance-An analysis, Solar Energy Materials and Solar Cells, 101, 36-45, 2012.

[6] Y.S.P. Ekdunge, and D. Simonsson, Proton conductivity of Nafion 117 as measured by Four-electrode AC impedance, The Journal of Electrochemical Society, 143, 4, 1254-1259, 1996.

[7] Volker Zagolla, Eric Tremblay, and Christophe Moser, Proof of principal demonstration of a self-tracking concentrator, Optics Express, Vol. 22, Issue S2, pp. A498-A510, 2014.

[8] T. Lopes, L. Andrade, H.A. Ribeiro, A. Mendes, Characterisation of photoelectrochemical cell for water splitting by electrochemical impedance spectroscopy, International Journal of Hydrogen Energy, 35, 2010.

[9] R. N. Pandey, K. S. Chandra Babu, and O. N. Srivastava, High conversion efficiency photoelectrochemical cells, Surface science, 52, pp. 125-192, 1996.

[10] C. 4.3b, Comsol Inc., 2013.

[11] R K Shevgaonkar, Electromagnetic waves, Tata McGraw Hill-Education, 2005.

[12] S. M. Sze, Physics of semiconductor devices, Wiley-interscience, 1969.

[13] F.P. Incropera, Principles of heat and mass transfer, John Wiley \& Sons, Inc., 2012.

[14] A. J. Bard and L. R. Faulkner, Electrochemical methods -Fundamentals and applications, John Wiley \& Sons, NewYork, 2nd edn, 2000. 
IHTC15-9526

[15] D. Nield and A. Bejan, Convection in Porous Media, 3rd ed., Springer, 2006.

[16] M. Le Bars and M.G. Worster, Interfacial Conditions Between a Pure Fluid and a Porous Medium: Implications for Binary Alloy Solidification, Journal of Fluid Mechanics, vol. 550, pp. 149-173, 2006.

[17] Bird, R.B.; Stewart, W.E.; Lightfoot, E.N. Transport Phenomena (2 ed.), Wiley, 2007.

[18] C.F. Curtiss and R.B. Bird, "Multicomponent Diffusion," Ind. Chem. Res., vol. 38 pp. 2515-2522, 1999.

[19] B. Van Zeghbroeck, Principles of Semiconductor Devices, 2011.

[20] N. D. Arora, J. R. Hauser, and D. J. Roulston, Electron and Hole mobilities in Silicon as a function of concentration and temperature, IEEE Trans. Electron Dev., ED-2(2),292.-295,1982.

[21] Y. Sone, P. Ekdunge and D. Simonsson, Journal of The Electrochemical Society, vol. 143, pp. 1254-1259, 1996.

[22] H. Ito, T. Maeda, A. Nakano and H. Takenaka, International Journal of Hydrogen Energy, vol. 36, pp. 739-746, 1966.

[23] K. Kinoshita, Electrochemical Oxygen Technology, John Wiley and Sons, Inc., 1992.

[24] L. Kriksunov, L. Bunakova, S. Zabusova and L. Krishtalik, Electrochimica Acta, vol. 39, pp. 137-142, 1994.

[25] A. Damjanovic, A. Dey and J. O. Bockris, Journal of The Electrochemical Society, vol. 113, pp. 739-746, 1966.

[26] N. M. Markovic, B. N. Grgur and P. N. Ross, The Journal of Physical Chemistry B, vol. 101, pp. 5405-5413, 1997.

[27] W. Sheng, H. A. Gasteiger and Y. Shao-Horn, Journal of The Electrochemical Society, vol. 157, pp. B1529-1536, 2010.

[28] Thermophysical Properties of Fluid Systems, NIST Web Book of Chemistry.

[29] M.J. Woodman, The thermal conductivity and electrical resistivity of Platinum. pp. 132-132, 1966. Platinum Metals Rev. 